\title{
RECENT RESULTS IN CHARM DECAYS FROM MARK III"
}

\author{
Jonathan F. Labs \\ Stanford Linear Accelerator Center, \\ Stanford University, Stanford, CA 94309 USA \\ Representing the \\ Mark III Collaboration
}

\begin{abstract}
Measurements of $D^{0} \rightarrow \overrightarrow{K^{0}} \pi^{0}, \bar{K}^{0} \eta$ and $\overrightarrow{K^{0}} \eta^{\prime}$ are reported. Preliminary results of a search for the doubly Cabibbo-suppressed decays $D^{+} \rightarrow K^{+} \pi^{-} \pi^{+}$and $K^{+} \pi^{0}$ are also given. Both analyses used $9.3 \pm 0.47 \mathrm{pb}^{-1}$ of data collected near the $\psi(3770)$ at SPEAR.
\end{abstract}

ANALYSIS OF $D^{0} \rightarrow \bar{K}^{0} \pi^{0}, \bar{K}^{0} \eta$ AND $\bar{K}^{0} \eta^{\prime}$

The decays, $D^{0} \rightarrow \overline{K^{0}} \pi^{0}, \bar{K}^{0} \eta$ and $\overline{K^{0}} \eta^{\prime}$ are interesting first in that they are color-suppressed. Secondly, the standard spectator diagrams only produce $\bar{K}^{0} \eta$ and $\bar{K}^{0} \eta^{\prime}$ through non-s $\bar{s}$ components in their wave functions; hence they should be produced with roughly equal strength. Non-spectator processes, such as W-exchange or final state interactions, can generate these channels via the $s \bar{s}$ components; these processes would cause an excess of $\bar{K}^{0} \eta^{\prime}$ over $\bar{K}^{0} \eta{ }^{[1]}$

Table I. ${ }^{[2]}$

\begin{tabular}{|c|c|c|c|}
\hline Mode & ARGUS & CLEO & MARKIII \\
\hline$D^{0} \rightarrow \overline{K^{0}} \pi^{0}$ & $1.7 \pm 0.4 \pm 0.3$ & $2.3 \pm 0.4 \pm 0.5$ & $1.8 \pm 0.2 \pm 0.2$ \\
$D^{0} \rightarrow \overline{K^{0}} \eta$ & $1.4 \pm 0.5 \pm 0.3$ & & $1.6 \pm 0.6 \pm 0.4$ \\
$D^{0} \rightarrow \overline{K^{0}} \eta^{\prime}$ & $1.9 \pm 0.4 \pm 0.3$ & & $3.3 \pm 0.3 \pm 1.0$ \\
\hline
\end{tabular}

Table 1. lists our measurements for each of these channels, together with ARGUS and CLEO measurements. None of the color-suppressed channels are greatly suppressed. Taking the Mark III and Argus numbers, the ratio $\mathrm{BR}\left(D^{0} \rightarrow \overline{K^{0}} \eta^{\prime}\right) / \mathrm{BR}\left(D^{0} \rightarrow \overline{K^{0}} \eta\right)$ is found to be $1.8 \pm 0.5$ (weighted average). Factoring out the phase space contributions, the ratio of matrix elements squared is $2.5 \pm 0.7$. That this number deviates from unity is an indication that non-spectator processes may play a role in hadronic charm decays.

\section{ANALYSIS OF DOUBLY CABIBBO-SUPPRESSED DECAYS}

Naively, the spectator model predicts doubly Cabibbo-suppressed decays (DCSD) to be suppressed relative to Cabibbo-allowed decays (CAD) by a factor of $\left(\rho^{2} \simeq 1\right) \tan ^{4} \theta_{C}$. Weak decay phenomenology, however, predicts $\rho^{2}>1$ for many DCSD channels. ${ }^{[3]}$ The $D^{+}$DCSD are interesting first becaure $\rho^{2} \gg 1$ is predicted. This enhancement arises from Pauli interference

* This work was supported in part by the Department of Energy, under contracts DE-AC03-76SF00515, DEAC02-76ER01195, DE-AC03-81ER40050,DE-AM03-76SF00010, and DE-AC02-87ER40318, and by the National Science Foundation.

Presented at the XII International Conference on Particles and Nuclei, Cambridge, Ma, June 25 - 29, 1990 
affecting CAD's but not DCSD's. Secondly, the $D^{+}$DCSD produce unique final states, unlike the $D^{0}$ DCSD which generate final states identical to those produced by $D^{0} \bar{D}^{0}$ mixing. Reported here are the preliminary results of the first search for $D^{+} \rightarrow K^{+} \pi^{-} \pi^{+}$and $K^{+} \pi^{0}$.

For the $K^{+} \pi^{-} \pi^{+}$analysis, proper track selection and anti-background requirements are first imposed. A scatter plot of the invariant mass vs, beam constrained mass is then formed from the resulting $\mathrm{K}^{+} \pi^{-} \pi^{+}$candidates. The beam constrained mass depends upon the correct track combination, while the invariant mass requires the right particle identification. Figure 1 shows the resulting plot where three events remain in the signal region. Monte Carlo studies estimate the background to be $0.8 \pm 0.3 \pm 0.3$ events in the signal region. The calculation of Ref. 3 predicts the number of expected events to be between 0.5 and 0.8 .

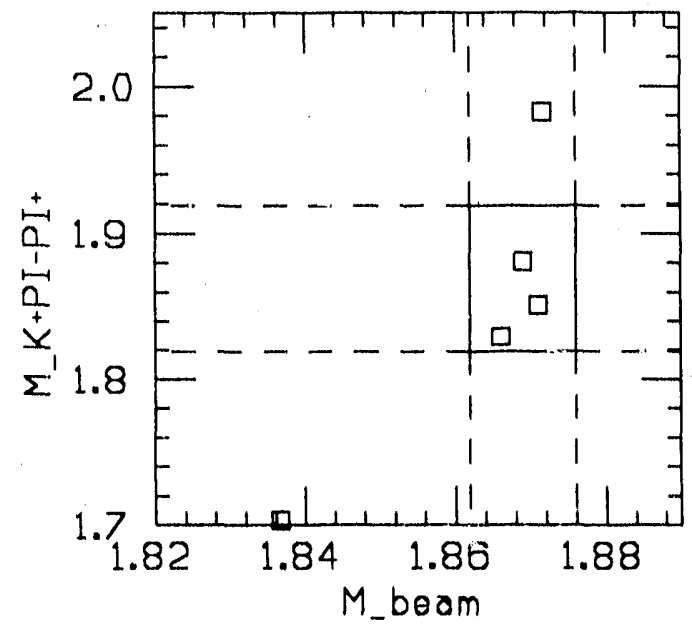

Fig. 1. $D^{+} \rightarrow K^{+} \pi^{-} \pi^{+}$candidates.

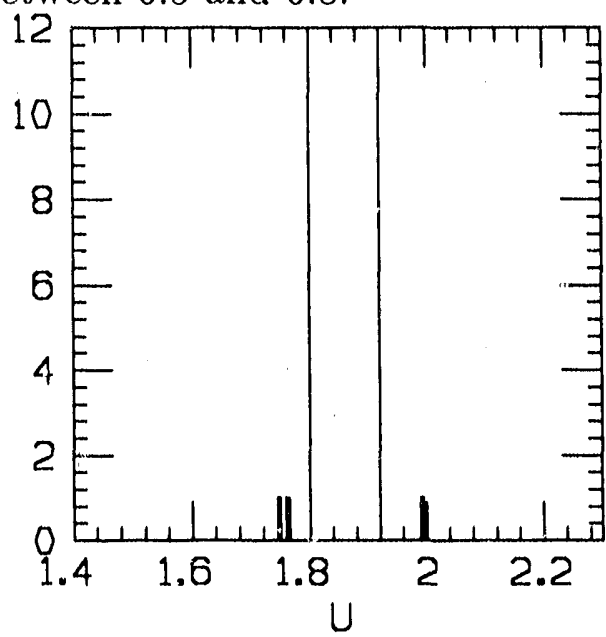

Fig. 2. $D^{+} \rightarrow K^{+} \pi^{0}$ candidates.

For the $K^{+} \pi^{0}$ analysis, proper track selection and anti-background requirements are imposed. A histogram of $U \equiv P_{\text {recoil }}^{\mu} P_{K \mu}$ is then made from the resulting $K^{+} \pi^{0}$ candidates. The $\mathrm{U}$ variable depends upon the correct track identification of the charged track. Figure 2 shows the resulting plot with no events remaining in the signal region. Monte Carlo studies estimate the level of background to be $<0.2$ at $90 \%$ CL. The number of real events expected ${ }^{[3]}$ is $\sim 0.2$.

A previous analysis of $D^{0} \bar{D}^{C}$ mixing and $D^{0}$ DCSD suggests that $\rho^{2}$ for the $D^{0}$ is larger than theoretical predictions ${ }^{[4]}$. The results for $D^{+} \rightarrow K^{+} \pi^{-} \pi^{+}$suggests a similar trend in the $\mathrm{I}^{+}$ DCSD.

\section{REFERENCES}

1. H. Lipkin, Phys. Rev. Lett., 46, 1307 (1981).

2. P. Karchin, In Proceedings of the 1989 Int. Symp. on Lepton and Photon Interactions at High Energies, Stanford, p. 105.

3. I. I. Bigi, In Proceedings of the Sixteenth SLAC Summer Institute on Particle Physics, Stanford, p. 31.

4. G. Gladding, In Proceedings of the Int. Symp. on Production and Decay of Heavy Flavors, Stanford, p. 178. 

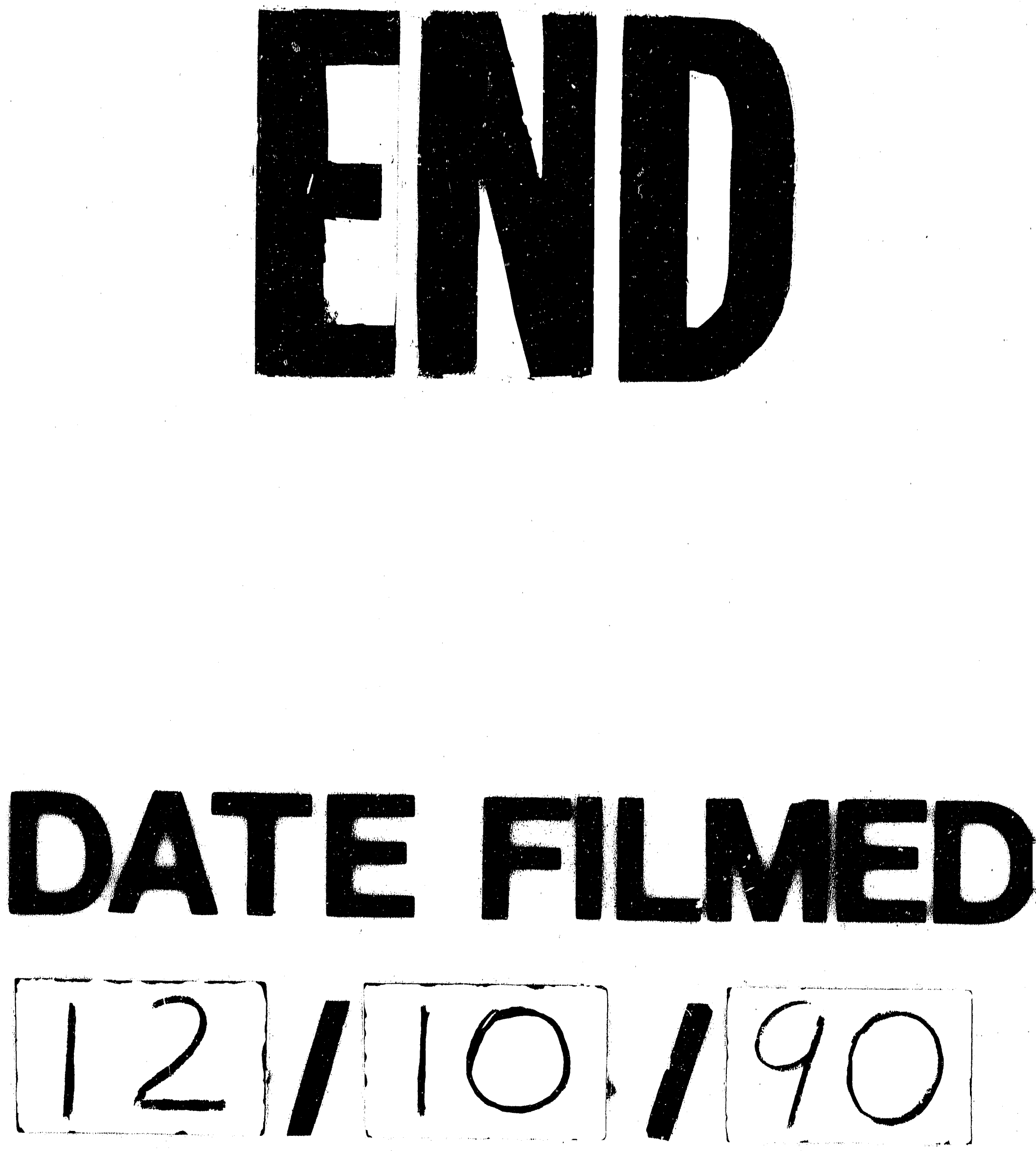
